\title{
REMOTE SENSING OBSERVATIONS AND NUMERICAL SIMULATION FOR MARTIAN LAYERED EJECTA CRATERS
}

\author{
L. $\mathrm{Li}^{1}{ }^{1}$, Z. Yue ${ }^{2}$, C. Zhang ${ }^{1}, \mathrm{D} \mathrm{Li}^{1}$ \\ ${ }^{1}$ College of Geomatics Science and Technology, Xi'an University of Science and Technology, Xi'an, China - lili330330@ sina.com, \\ zhchunsen@aliyun.com, lidan0101miao@sina.com \\ ${ }^{2}$ Institute of Remote Sensing and Digital Earth, Chinese Academy of Sciences, Beijing, China - yuezy@ radi.ac.cn
}

\section{Commission III, ICWG III/II}

KEY WORDS: Martian Surface, Impact Crater, Remote Sensing, Morphological Characteristic, Numerical Simulation

\begin{abstract}
:
To understand past Martian climates, it is important to know the distribution and nature of water ice on Mars. Impact craters are widely used ubiquitous indicators for the presence of subsurface water or ice on Mars. Remote sensing observations and numerical simulation are powerful tools for investigating morphological and topographic features on planetary surfaces, and we can use the morphology of layered ejecta craters and hydrocode modeling to constrain possible layering and impact environments. The approach of this work consists of three stages: Firstly, the morphological characteristics of the Martian layered ejecta craters are performed based on Martian images and DEM data. Secondly, numerical modeling layered ejecta are performed through the hydrocode iSALE (impact-SALE). We present hydrocode modeling of impacts onto targets with a single icy layer within an otherwise uniform basalt crust to quantify the effects of subsurface $\mathrm{H}_{2} \mathrm{O}$ on observable layered ejecta morphologies. The model setup is based on a layered target made up of a regolithic layer (described by the basalt ANEOS), on top an ice layer (described by ANEOS equation of $\mathrm{H}_{2} \mathrm{O}$ ice), in turn on top of an underlying basaltic crust. The bolide is a $0.8 \mathrm{~km}$ diameter basaltic asteroid hitting the Martian surface vertically at a velocity of $12.8 \mathrm{~km} / \mathrm{s}$. Finally, the numerical results are compared with the MOLA DEM profile in order to analyze the formation mechanism of Martian layered ejecta craters. Our simulations suggest that the presence of an icy layer significantly modifies the cratering mechanics, and many of the unusual features of SLE craters may be explained by the presence of icy layers. Impact cratering on icy satellites is significantly affected by the presence of subsurface $\mathrm{H}_{2} \mathrm{O}$.
\end{abstract}

\section{INTRODUCTION}

One of the most important points of Mars research consists in understanding the evolution of water on its surface and subsurface. The conventional picture of Martian mid-latitude ice has been that it is young and pore-filling ground ice, which responds quickly to varying orbital and climatic conditions via atmospheric exchange (Bryson et al., 2008, Hudson et al., 2009). Widespread near-surface ice deposits are inferred on Mars. Iceexposing impacts (Dundas et al., 2014) and thermokarstic expansion of secondary craters (Viola et al., 2015) suggest ice in the northern mid-latitudes of Mars is in the form of relatively pure, excess ice (higher water ice abundances than available in the pore spaces of the regolith).

Impact craters are widely distributed on most terrestrial planets, and they play a major role in shaping planetary surfaces and elucidating the climatic and geologic history of planets. Impact craters are widely used ubiquitous indicators for the presence of subsurface water or ice on Mars. Martian impact craters typically possess distinct layered ejecta blankets, consisting of one or more layers of material emplaced by fluidization processes that differ significantly from their lunar and Mercurian counterparts (Carr et al., 1977). Martian layered ejecta craters have been classified into different types including single-layered ejecta (SLE) craters (Fig.1), double-layered ejecta (DLE) craters, and multiple-layered ejecta (MLE) craters (Barlow et al., 2000). The mode of emplacement of Martian layered morphologies has been a subject of intense study over the last few years. Observational data, laboratory experiments, and numerical modeling have provided important information about Martian layered ejecta structures. Martian layered ejecta craters are thought to form by impacts onto ice/water-rich areas in the aquifer of Mars (Mouginis-Mark, 1978, Reiss et al., 2005, Senft and Stewart, 2008, Kadish and Head, 2011).

The geometry of layered ejecta deposits is important for understanding the mode of emplacement of layered ejecta. The morphology of crater ejecta blankets on Mars have been shown to provide a good qualitative indicator for the presence or absence of subsurface liquid water or ice in the impacted target region (Barlow and Perez, 2003). Analyses of the global distribution and detailed morphology of layered ejecta craters are valuable for investigating the ejecta emplacement processes, as well as searching for spatial and/or temporal variations in ejecta characteristics that may be caused by latitude, altitude, or the properties of the target material. As we have shown in previous work, we perform a global analysis of the morphologies of SLE, DLE, and MLE craters, including their locations, dimensions, ejecta mobility, lobateness ( $\mathrm{Li}$ et al., 2015). Our survey of the distribution and characteristics of layered ejecta craters reveals that strong correlations exist between ejecta mobility and latitude, which provides more detailed information and insights of connection between the layered ejecta morphologies and the subsurface volatiles.

\footnotetext{
* Corresponding author
} 


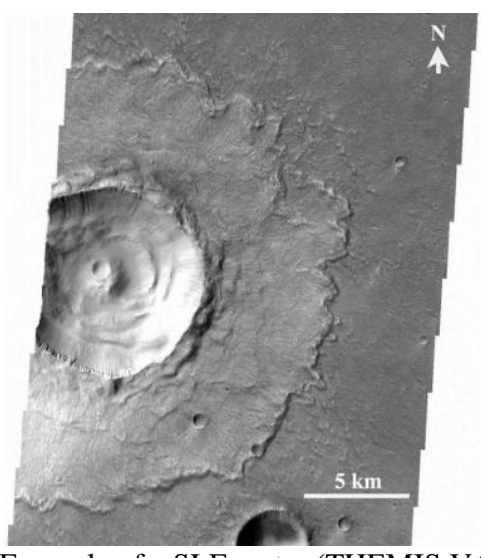

Figure 1. Example of a SLE crater (THEMIS V45965012)

Numerical modeling is a fundamental tool for understanding of the dynamics of impact cratering, especially at planetary scales. Numerical studies of impacts into marine targets displayed that morphologic variations are dependent upon the thickness of the water layer and the size of the impactor (Ormo et al., 2004). Numerical experiments have shown significant changes in crater and ejecta blanket morphologies with strength contrasts in the target. Strength layers modify ejection angles, and a target with weaker layers will undergo more collapse, resulting in shallower craters with terraces and benches (Senft and Stewart, 2007). However, much work remains to be done on the general problem of how icy layers in the target affect crater and ejecta blanket morphologies at the planetary scale. Understanding the observable features from cratering in icy, layered surfaces may lead to inferences about subsurface structures on many bodies in the solar system.

In this research, we present numerical simulations of crater formation under Martian conditions with a single near-surface icy layer to investigate changes in crater morphology. We conduct simulations of impacts onto targets with a single icy layer within an otherwise uniform basalt crust. We aim at presenting the numerical modeling of the formation of SLE craters, which may allow us to place additional constraints on the finer subsurface structure that leads to this peculiar morphology.

\section{METHODOLOGY}

We integrated Thermal Emission Imaging System (THEMIS) images (100 m/pixel) and Mars Orbiter Laser Altimeter (MOLA) data $(463 \mathrm{~m} /$ pixel) in ArcGIS software to manually locate all visible layered ejecta craters. After crater identification, we extracted the characteristic parameters for each SLE crater. The basic characteristic parameters of SLE craters involved diameter, depth, ejecta mobility $(\mathrm{EM})$, lobateness $(\Gamma)$, onset diameter $(\mathrm{Dr})$, and depth/diameter ratio (d/D).

Cratering simulations are conducted through the hydrocode iSALE. Initially developed by Amsden (Amsden et al., 1980), the code has been enhanced through modifications which include an elasto-plastic constitutive model, fragmentation models, various equations of state (EoS), multiple materials, a novel porosity compaction model, the $\varepsilon$ - $\alpha$-model (Melosh et al., 1992, Ivanov et al., 1997, Collins et al., 2004, Wunnemann et al., 2006). In addition, the code is well tested against laboratory experiments at low and high strain-rates (Wunnemann et al., 2006) and other hydrocodes (Pierazzo et al., 2008).
Numerical modeling provides a method of studying the physics of impact cratering at all scales using a set of differential equations established through the principles of conservation of momentum, mass and energy from a macroscopic point of view. Hydrocode iSALE includes an Equation of State (EoS) governing the bulk thermodynamic material response, and a constitutive model describing the response of a material to stresses that induce deviatoric deformations of changes of shape. Material models are a crucial component of hydrocode simulations (Pierazzo et al., 2005). An accurate material model especially related to the constitutive model and equation of state, thus, particular care should applied to these areas.

\subsection{Constitutive Model}

The strength model describes the response of a material to deviatoric stresses. We use the strength model developed by Collins et al. (2004). In this model, shear strength is linearly degraded from an intact strength value (strength controlled by the creation of new fractures) to a fragmented (strength controlled by friction) value. A dimensionless scalar variable called damage is introduced to track this degradation; completely intact rock has a damage of zero, and completely fragmented rock has a damage of one. Thus, shear strength is a function of damage, temperature, and pressure, and tensile strength is a function of damage. The rock strength model defines the yield strength $Y$ according to Eq. (1):

$$
Y=Y_{d} D+Y_{i}(1-D)
$$

where $D$ is a scalar measure of damage ( $0=$ intact; $1=$ damaged $)$ that is computed by one of the damage models. The damaged material strength, $Y_{d}$ is defined by Eq. (2):

$$
Y_{d}=\min \left(Y_{d 0}+\mu_{d} p, Y_{d m}\right)
$$

where $p$ is pressure.

The IVANOV damage model (Ivanov et al., 1997) prescribes damage $D$ as a function of plastic strain according to Eq. (3):

$$
D=\min \left(\frac{\varepsilon_{p}}{\varepsilon_{f}}, 1\right)
$$

where $\varepsilon_{p}$ is an invariant measure of the accumulated total plastic strain (always positive) and $\varepsilon_{f}$ is the plastic strain at failure.

We use the thermal softening model developed by Ohnaka and Mitiyasu (1995). In this model, the shear strength of rock materials depends on temperature, reducing to zero at the melting temperature. ISALE approximates this behavior using the simple relationship according to Eq. (4):

$$
Y=Y_{c} \tanh \left(\xi\left(\frac{T m}{T}-1\right)\right)
$$

where $Y_{c}$ is the cold yield strength (including the effect of damage), $Y$ is the thermally softened strength, $\mathrm{T}$ is temperature, $T_{m}$ is the melt temperature and $\xi$ is a material constant.

In this research, we use porosity model parameters of Wunnemann et al. (2006), which comprises four regimes that describe the compression of a pristine porous material. ISALE calculates the thermodynamic state of a porous material by 
separating the compaction of pore space from the compression of the solid component. The pressure in the porous material is given by the pressure in the solid material divided by the distension according to Eq. (5):

$$
p=\frac{1}{\alpha} p_{s}\left(\rho_{s}, E\right)
$$

where the pressure $p$ in a porous material is a function of the bulk density $\rho$, internal energy $E$, and distension $\alpha$, which is the ratio of the density of the solid material $\rho_{s}$ to the bulk density.

The strength-damage model described above will not reproduce the morphology of craters above the simple to complex transition, for which an additional weakening mechanism is needed. Here we use acoustic fluidization (Collins, 2002) to aid complex crater collapse, and in the absence of better information, we assume that all materials have the same acoustic fluidization parameters. This assumption is supported by work by Bray et al. (Bray et al., 2008), who show that similar acoustic fluidization parameters for ice and rock reproduce the morphology of central peak craters on the moon and Ganymede.

\subsection{Equation of State}

The equation of state describes the material's thermodynamic state over a wide range of pressures, temperatures, and specific volumes and it is critical in the early stages of the impact (Pierazzo and Collins, 2003). For each material an equation of state (EOS) must be defined. Hydrocode iSALE supports two EOS-types, data tables produced by ANEOS, and parameters defining the Tillotson analytical EOS.

Basalt is by far the most abundant igneous rock on Earth's surface, and it is also abundant on the Moon, Mars, and Venus (Pierazzo et al., 2005). In this research, ANEOS tabular equations of state for basalt (Pierazzo et al., 2005) and $\mathrm{H}_{2} \mathrm{O}$ (Ivanov, 2005) are used to describe the pressure, density, and temperature properties of the materials. Following Senft and Stewart (2008), we model rock and $\mathrm{H}_{2} \mathrm{O}$ water/ice as separate target materials. This has been done by adding $\mathrm{H}_{2} \mathrm{O}$ as individual layers that are explicitly resolved in the computational grid.

\subsection{Description of Simulations}

To investigate the effect of icy layers in the target on the cratering process, we conduct simulations of impacts onto a target with a single icy layer of varying thickness and ice content and at varying depths. The bolide is a $0.8 \mathrm{~km}$ diameter basaltic asteroid hitting the Martian surface vertically at a velocity of $12.8 \mathrm{~km} / \mathrm{s}$ (median impact velocity of asteroids on Mars (Senft and Stewart, 2008)). Our simulations model impact angles of $90^{\circ}$ from the surface in the Martian gravity field of $3.71 \mathrm{~m} / \mathrm{s}^{2}$. The projectile is kept resolved to $20 \mathrm{~m}$. A spatial resolution of 20 cells per projectile radius (CPPR) is maintained around the impact point, followed by regions of progressively lower resolution.

All calculations are initialized with a Martian crust under lithostatic pressure and with a geothermal temperature gradient of $15 \mathrm{~K} / \mathrm{km}$, consistent with estimates by Clifford (Clifford, 1993). The pressure and temperature at the surface are assumed to be 6 mbar and $210 \mathrm{~K}$, respectively, to be broadly representative of the present epoch on Mars. These model parameters as shown in Table 1 were derived after testing a variety of properties of the target layers, as well as projectile size and speed.

\begin{tabular}{|c|c|c|}
\hline \multirow{4}{*}{$\begin{array}{c}\text { Basic } \\
\text { parameters }\end{array}$} & gravity & $3.71 \mathrm{~m} / \mathrm{s}^{2}$ \\
\cline { 2 - 3 } & surface temperature & $210 \mathrm{~K}$ \\
\cline { 2 - 3 } & temperature gradient & $15 \mathrm{~K} / \mathrm{km}$ \\
\cline { 2 - 3 } & surface pressure & $6 \mathrm{mbar}$ \\
\hline \multirow{4}{*}{$\begin{array}{c}\text { Projectile } \\
\text { parameters }\end{array}$} & velocity & $12.8 \mathrm{~km} / \mathrm{s}$ \\
\cline { 2 - 3 } & material & basalt \\
\cline { 2 - 3 } & type & spheroid \\
\cline { 2 - 3 } & radius & $0.4 \mathrm{~km}$ \\
\hline Target & CPPR & 20 \\
\hline parameters & layers number & 3 \\
\cline { 2 - 3 } & layer material & basalt $/ \mathrm{H}_{2} \mathrm{O} /$ basalt \\
\hline
\end{tabular}

Table 1. The parameters of iSALE simulations

All calculations are conducted in 2D under cylindrical symmetry. As shown in Figure 2, the model setup is based on a layered target made up of a regolithic layer (described by the basalt ANEOS), on top an ice layer (described by ANEOS equation of $\mathrm{H}_{2} \mathrm{O}$ ice), in turn on top of an underlying basaltic crust. In all simulations, the icy layers are kept resolved to at least 10 cells across a layer.

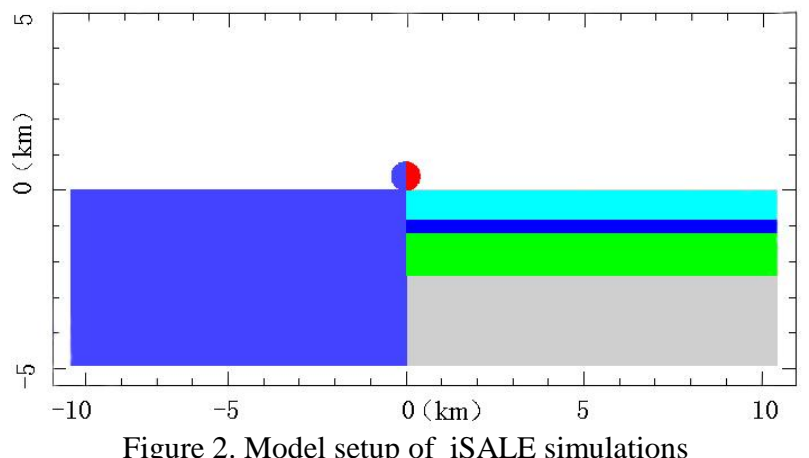

Each simulation was extended long enough to allow the shock wave propagating in the target to decay well below typical melting thresholds of rocks (20 GPa (Senft and Stewart, 2008)). Lagrangian tracer particles are used to track the history of parcels of material as they are advected through the Eulerian mesh. The simulations model the well-known sequence of crater formation: initial penetration of the projectile, transient cavity growth, and crater gravity collapse. Finally, the results of the models are then compared with the MOLA DEM profile in order to derive the properties which best explain the crater morphology.

\section{RESULTS AND DISCUSSIONS}

In our previous work, we mapped the locations of 7,781 SLE craters using THEMIS images and MOLA data across the entire Martian surface, statistical results show that there are minor concentrations of SLE craters in the regions of Tharsis Montes (Li et al., 2015). In this research, a typical middle-size SLE craters (Fig. 3) in Tharsis Montes has been considered for analysis.

To investigate the effect of icy layers in the target on the cratering process, we conduct simulations of impacts onto a target with a single icy layer of varying thickness and ice content and at varying depths. The formation hypothesis of the SLE crater may be tested by detailed comparison between 
impact cratering simulations and measurements of crater forms using topography data set.

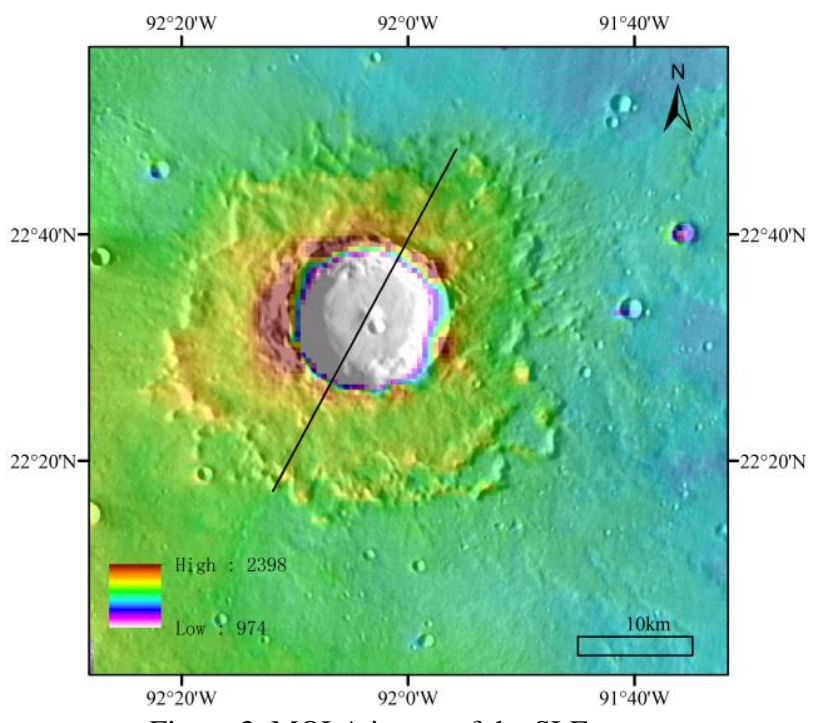

Figure 3. MOLA image of the SLE crater

\subsection{Crater Morphology}

A single icy layer significantly modifies cratering mechanics and can result in a range of observable effects on final crater morphology, depending upon the burial depth and thickness of the layer relative to the size of the impactor. A model case (Fig. 4) presents time series from simulations of a $0.8 \mathrm{~km}$ diameter projectile impacting onto the Martian surface.
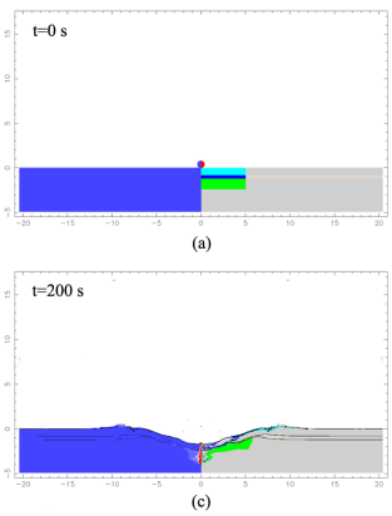

Figure 4. Projectile impacting at $12.8 \mathrm{~km} / \mathrm{s}$ for layered ice basalt target under Martian gravity

In Fig. 4a, the target is a buried ice layer (ice thickness $0.4 \mathrm{~km}$, and ice burial depth $0.8 \mathrm{~km}$ ), i.e., we do not attempt, at this stage, to use mixed cells target (where material mixing occurs at a sub-cell scale) or wet basalt target (complete homogenization based on mass-weighted, average properties of the two materials, basalt and water). A buried ice layer causes the top basalt layer to tear away from the underlying material at early times $(100 \mathrm{~s})$, shock wave reflections between the layers increase the fragmentation of the overlying basalt and modify the ejecta trajectories (Fig. 4b). As the ice being extruded from all sides of the crater meets, it creates a central uplift (Fig. 4c) which later collapses back down in on itself and flows outward (Fig. 4d).

The assumption of alternating intact ice and basalt layers is oversimplified; however, these simulations illustrate the range of possible outcomes of cratering into a target with icy layers. A buried ice layer displays modification of the ejecta trajectories due to wave reflections as well as a late stage icy extrusion from the crater wall into the floor. The results of this investigation suggest that a 3-layer target is needed to account for both the crater morphology and layered ejecta blankets.

While the magnitude and type of effects produced by an icy layer will depend upon a number of complex factors (burial depth, thickness, ice content of layer, etc.), in general, we conclude that significant effects (such as late stage icy extrusions) are seen when the thickness of the icy layer is at least $30 \%$ of the diameter of the projectile.

\subsection{Comparison with Observations}

Numerical modeling is a powerful tool for investigating the formation of large impact craters but is one that must be validated with observational evidence. From MOLA data of the SLE crater (Fig. 3), we took profiles across the crater to compare to our numerical models. As shown in Figure 5 and Table 2, simulation results are compared to observations of Martian impact craters.

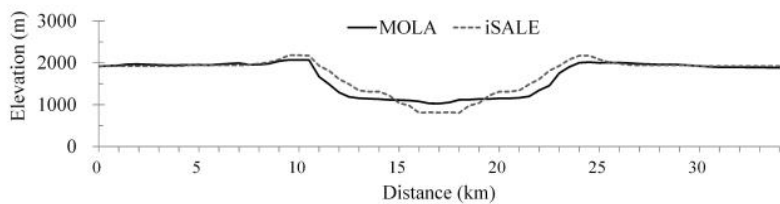

Figure 5. Profiles of the crater final stage (iSALE 2D) compared to the MOLA data

Observable features demonstrated by the modeling include variations in crater morphometry and icy infill of the crater floor during the late stages of crater formation. The good fit between models and observations will allow us to understand the surficial layering and composition of specific regions of Mars.

\begin{tabular}{|c|c|c|}
\hline Parameters & MOLA & iSALE \\
\hline Diameter $(\mathrm{km})$ & 13.30 & 12.20 \\
\hline depth $(\mathrm{km})$ & 0.99 & 1.12 \\
\hline depth/Diameter ratio (d/D) & 0.074 & 0.092 \\
\hline ejecta extent $(\mathrm{km})$ & 12.80 & 13.20 \\
\hline $\mathrm{H}_{2} \mathrm{O}$ vol\% & None & $12.3 \%$ \\
\hline
\end{tabular}

Table 2. Comparison between the results of observations and iSALE simulations

The parameters comparison between the results of remote sensing observations and numerical simulation infer that many of the unusual features of Martian SLE craters may be explained by the presence of icy layers in the target. The best fit of this SLE crater with the DEM was obtained with $0.8 \mathrm{~km}$ diameter projectile impacting at $12.8 \mathrm{~km} / \mathrm{s}$ for layered ice basalt target (ice thickness $0.4 \mathrm{~km}$, and ice burial depth $0.8 \mathrm{~km}$ ).

Understanding the conditions that formed this thick, extensive but heterogeneous layer of ice will improve our understanding of the Martian climate system. Our preliminary simulation results suggest that many of the anomalous features associated with Martian SLE craters, including variations in ejecta blanket morphologies, may be a result of icy layers. 


\section{CONCLUSIONS}

We present a multi-faceted study of evidence for subsurface layering of ice associated with Martian SLE craters. Using the iSALE hydrocode, coupled to the ANEOS equation of state for basalt and ice, we create high-resolution two-dimensional impact models. Our preliminary results suggest that icy layers modify the excavation processes and leading to significant variations in the ejecta blankets, a 3-layer target is needed to account for both the crater morphology and layered ejecta blankets. Impact cratering on icy satellites is significantly affected by the presence of subsurface $\mathrm{H}_{2} \mathrm{O}$.

The target layer composition and geometries investigated in this paper are simplified representations of the near-surface crust on Mars. While this work has demonstrated a wide range of observable features resulting from ice-rich layers, more detailed studies and observational constraints are needed to concretely link the modeling and observations.

\section{ACKNOWLEDGEMENTS}

We gratefully acknowledge the developers of iSALE-2D, including G. Collins, K. Wunnemann, D. Elbeshausen, B. A. Ivanov and J. Melosh (www.iSALE-code.de). This study was supported by the Foundation of XUST under Grant NO. 6310217015 and 6310117012 .

\section{REFERENCES}

Amsden, A. A., H. M. Ruppel and C. W. Hirt, 1980. SALE: A simplified ALE computer program for fluid flow at all speeds. Report LA-8095, Los Alamos Scientific Lab, New Mexcio, USA.

Barlow, N. G., J. M. Boyce, F. M. Costard, R. A. Craddock, J. B. Garvin, S. E. H. Sakimoto, R. O. Kuzmin, D. J. Roddy and L. A. Soderblom, 2000. Standardizing the nomenclature of Martian impact crater ejecta morphologies. Journal of Geophysical Research: Planets, 105(E11), pp. 26733-26738.

Barlow, N. G. and C. B. Perez, 2003. Martian impact crater ejecta morphologies as indicators of the distribution of subsurface volatiles. Journal of Geophysical Research-Planets, 108(E8).

Bray, V. J., G. S. Collins, J. V. Morgan and P. M. Schenk, 2008 The effect of target properties on crater morphology: Comparison of central peak craters on the Moon and Ganymede. Meteoritics \& Planetary Science, 43(12), pp. 1979-1992.

Bryson, K. L., V. Chevrier, D. W. G. Sears and R. Ulrich, 2008. Stability of ice on Mars and the water vapor diurnal cycle: Experimental study of the sublimation of ice through a finegrained basaltic regolith. Icarus, 196(2), pp. 446-458.

Clifford, S. M., 1993. A Model for the Hydrologic and Climatic Behavior of Water on Mars. Journal of Geophysical ResearchPlanets, 98(E6), pp. 10973-11016.

Collins, G. S., 2002. Numerical modelling of large impact crater collapse, Imperial College London.

Collins, G. S., H. J. Melosh and B. A. Ivanov, 2004. Modeling damage and deformation in impact simulations. Meteoritics \& Planetary Science, 39(2), pp. 217-231.
Dundas, C. M., S. Byrne, A. S. McEwen, M. T. Mellon, M. R. Kennedy, I. J. Daubar and L. Saper, 2014. HiRISE observations of new impact craters exposing Martian ground ice. Journal of Geophysical Research-Planets, 119(1), pp. 109-127.

Hudson, T. L., O. Aharonson and N. Schorghofer, 2009. Laboratory experiments and models of diffusive emplacement of ground ice on Mars. Journal of Geophysical ResearchPlanets, 114(E1).

Ivanov, B. A., 2005. Numerical modeling of the largest terrestrial meteorite craters. Solar System Research, 39(5), pp. 381-409.

Ivanov, B. A., D. Deniem and G. Neukum, 1997. Implementation of dynamic strength models into 2D hydrocodes: Applications for atmospheric breakup and impact cratering. International Journal of Impact Engineering, 20(1-5), pp. 411-430.

Kadish, S. J. and J. W. Head, 2011. Impacts into non-polar icerich paleodeposits on Mars: Excess ejecta craters, perched craters and pedestal craters as clues to Amazonian climate history. Icarus, 215(1), pp. 34-46.

Li, L., Z. Yue, K. Di and M. Peng, 2015. Observations of Martian layered ejecta craters and constraints on their formation mechanisms. Meteoritics \& Planetary Science, 50(3), pp. 508522 .

Melosh, H. J., E. V. Ryan and E. Asphaug, 1992. Dynamic fragmentation in impacts: Hydrocode simulation of laboratory impacts. Journal of Geophysical Research: Planets, 97(E9), pp. 14735-14759.

Mouginis-Mark, P. J., 1978. Morphology of Martian rampart craters. Nature, 272, pp. 691-694.

Ohnaka and Mitiyasu, 1995. A shear failure strength law of rock in the brittle-plastic transition regime. Geophysical Research Letters, 22(1), pp. 25-28.

Ormo, J., J. M. Dohm, J. C. Ferris, A. Lepinette and A. G. Fairen, 2004. Marine-target craters on Mars? An assessment study. Meteoritics \& Planetary Science, 39(2), pp. 333-346.

Pierazzo, E., N. Artemieva, E. Asphaug, E. Baldwin, J. Cazamias, R. Coker, G. Collins, D. Crawford, T. Davison and D. Elbeshausen, 2008. Validation of numerical codes for impact and explosion cratering: Impacts on strengthless and metal targets. Meteoritics \& Planetary Science, 43(12), pp. 19171938.

Pierazzo, E., N. Artemieva and B. Ivanov, 2005. Starting conditions for hydrothermal systems underneath Martian craters: Hydrocode modeling. Geological Society of America Special Papers, 384, pp. 443-457.

Pierazzo, E. and G. Collins, 2003. A brief introduction to hydrocode modeling of impact cratering. Cratering in marine environments and on ice. Berlin Heidelberg, Springer, pp. 323340 .

Reiss, D., E. Hauber, G. Michael and R. Jaumann, 2005. Small rampart craters in an equatorial region on Mars: Implications 
for near-surface water or ice. Geophysical research letters, 32(10).

Senft, L. E. and S. T. Stewart, 2007. Modeling impact cratering in layered surfaces. Journal of Geophysical Research: Planets, 112(E11).

Senft, L. E. and S. T. Stewart, 2008. Impact crater formation in icy layered terrains on Mars. Meteoritics \& Planetary Science, 43(12), pp. 1993-2013.

Viola, D., A. S. McEwen, C. M. Dundas and S. Byrne, 2015. Expanded secondary craters in the Arcadia Planitia region, Mars: Evidence for tens of Myr-old shallow subsurface ice. Icarus, 248, pp. 190-204.

Wunnemann, K., G. S. Collins and H. J. Melosh, 2006. A strain-based porosity model for use in hydrocode simulations of impacts and implications for transient crater growth in porous targets. Icarus, 180(2), pp. 514-527. 